\title{
Rádios Definidos por Software e GNU Radio aplicados aos cursos de Graduação em Engenharia Eletrônica
}

DOI: 10.37702/2175-957X.COBENGE.2021.3665

Marcílio André Félix Feitosa - marcilio@poli.br

Universidade de Pernambuco

Av. Recife 3856

50860-000 - Recife - PE

Luis Henrique Nunes da Silva - Ihns@poli.br

UPE

Rua Benfica 455

50720-001 - Recife - PE

Gustavo Oliveira Cavalcanti - gustavooc@poli.br

Universidade de Pernambuco

Rua Major Médico Fonseca de Matos 670

54440-370 - Jaboatão - PE

Paulo Hugo Espirito Santo Lima - paulo.hugo@poli.br

UPE

R. Benfica 455

50720-001 - Recife - PE

Resumo: Com a constante diminuição dos investimentos, pelos governantes, na manutenção e criação de novos laboratórios universitários, é necessário inovar. Esse trabalho propõe a criação de um laboratório de telecomunicações para o curso de graduação em eletrônica da Escola Politecnica da Universidade de Pernambuco. A utilização de Radios Definidos por Software mais simples e mais baratos, junto com a adoção de software livre para a configuração dos rádios e simulação dos sistemas, permite a criação de ambientes onde o aluno poderá aprender, na prática, técnicas de comunicações, antes ofertadas de forma puramente teórica. Aprender praticando auxilia na compreensao e na retenção dos conhecimentos. $O$ artigo sugere uma configuração mínima para o laboratório e apresenta uma estimativa orçamentária. Além disso, sugere diferentes aplicações que podem ser desenvolvidas nas diversas disciplinas, obrigatórias ou eletivas, ofertadas no curso. 


\section{(C) COBENGE

Palavras-chave: Radios Definidos por Software, GNU Radio, Engenharia Eletrônica, Laboratório de Telecomunicações 


\section{RADIOS DEFINIDOS POR SOFTWARE E GNU RADIO APLICADOS AOS CURSOS DE GRADUAÇÃO EM ENGENHARIA ELETRÔNICA}

\section{INTRODUÇÃO}

Com raras exceções, Universidades públicas Federais, Estaduais e Municipais, sofrem com a falta de investimentos em Laboratórios Didáticos de Ensino. Até nas Universidades privadas o investimento é baixo. Um levantamento realizado em $2018 \mathrm{com}$ base nos dados disponibilizados pelo MEC, informa os valores repassados pelo governo às Universidades Federais no período de 2010 a 2018 para gastos não obrigatórios (que não envolvem, por exemplo, a folha de pagamento) (MORENO, 2018 e FOLHA, 2019). Esse levantamento pode ser visto, de forma gráfica, na Figura 1. Através dele observa-se que, desde 2014, o orçamento para investimentos cai rapidamente a cada ano. O período do estudo foi até 2018, mas em 2019 tivemos mais uma queda, com o contingenciamento de $30 \%$ do montante disponível para o custeio e os investimentos dessas instituições, reduzindo a $\mathrm{R} \$ 5,2$ bilhões naquele ano. Não é preciso dizer que, em 2020, com o advento da pandemia mundial provocada pelo novo corona vírus, a situação apenas piorou. Além disso, o mesmo estudo indicou que, nesse mesmo período, o número de alunos ingressantes é sempre maior que o do ano anterior (MORENO, 2018).

Figura 1 - Repasses do governo às universidades federais.

Orçamento Previsto (círculos) e Valores Empenhados.

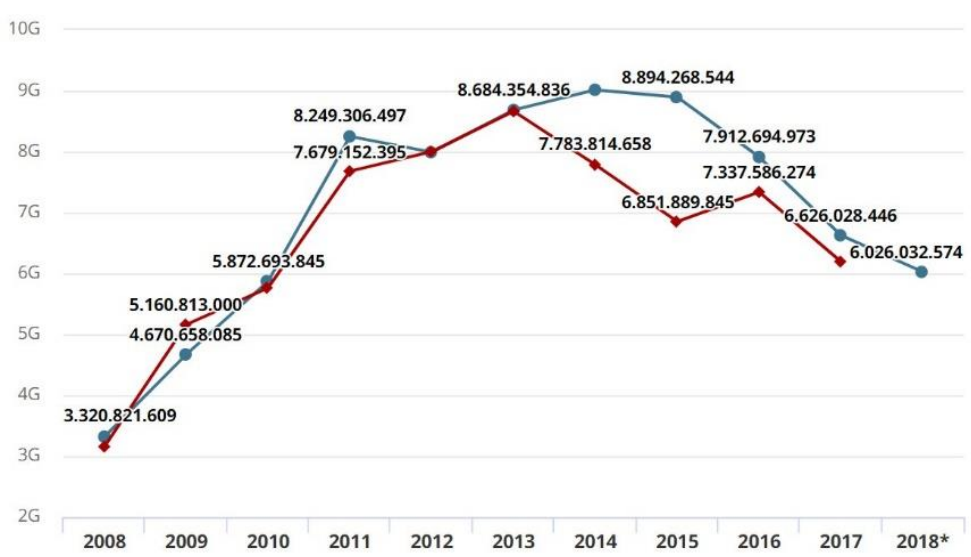

Fonte: Subsecretaria de Plan. e Orçamento/MEC (MORENO, 2018).

Nas Universidades Estaduais, como é o caso da Universidade de Pernambuco (UPE) onde foi realizado esse trabalho, a situação não é diferente. Como pode ser observado no gráfico da Figura 2, os investimentos do Governo do Estado na UPE sofreram uma queda de mais de 50\% na segunda metade da década analisada (2010 a 2019).

Entre 2010 e 2014 era investido, em média, 17 milhões de reais, enquanto de 2015 a 2019 só foi investido cerca de 8 milhões por ano. Os dados foram obtidos através do Portal da Transparência do Estado (http://web.transparencia.pe.gov.br/despesas/despesageral/), em Despesas Gerais, determinando como categoria econômica o item 4 (Despesas de Capital) e o grupo 4 (Investimentos). Nesse grupo estão inclusos gastos com obras, instalações, equipamentos e materiais permanentes. Em matéria recente do caderno Educação do jornal O Globo, nos últimos 17 anos, o ensino superior público vem crescendo e o número de alunos matriculados saltou de 574 mil para 1,3 milhão (ALFANO, 2021). Já 
a verba disponível para investimentos e manutenção das instituições em 2021 caiu ao patamar de 2004: $R \$ 2,5$ bilhões.

Figura 2 - Despesas de com Investimentos do Governo na UPE.

Despesas de Capital com Investimentos

(em milhões de reais)

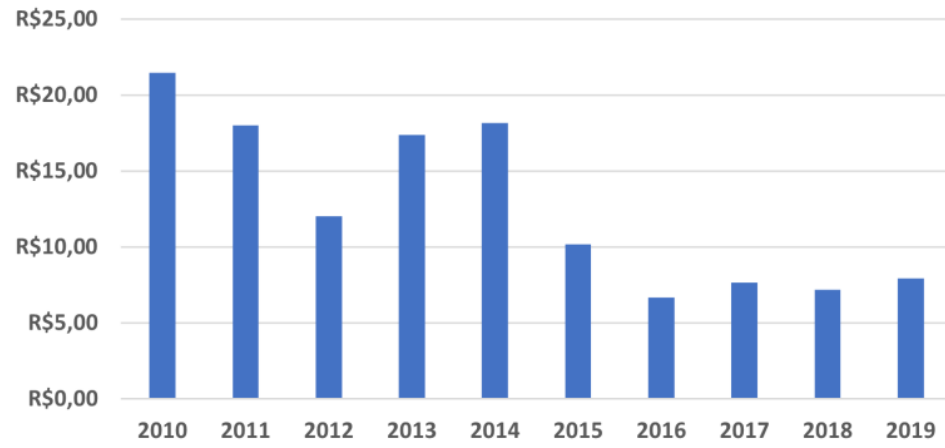

Fonte: http://web.transparencia.pe.gov.br/despesas/despesa-geral/.

Visto isso, é fácil perceber que a implantação de novos laboratórios didáticos de ensino para os cursos de graduação não tem sido uma prioridade orçamentária para os governos. Até mesmo a manutenção dos laboratórios existentes muitas vezes fica em segundo plano. As direções dos campi, bem como as coordenações dos cursos, fazem malabarismos tentando fornecer a melhor estrutura possível, mas, sem recursos, é uma tarefa difícil. Laboratórios de pesquisa são, na sua maioria, financiados por órgãos de apoio à pesquisa, como o CNPq (Conselho Nacional de Desenvolvimento Científico e Tecnológico), a FINEP (Financiadora de Estudos e Projetos) e a FACEPE (Fundação de Amparo à Ciência e Tecnologia de Pernambuco), mas apenas os alunos participantes da pesquisa, em geral, têm acesso aos equipamentos de ponta financiados por essas pesquisas.

Mas, como formar profissionais capacitados e competitivos sem laboratórios de graduação atualizados com o que se usa no mercado de trabalho? O Laboratório de Eletrônica da Escola Politécnica de Pernambuco teve sua última atualização em 2016, com a substituição de equipamentos obsoletos e defeituosos. Todos os osciloscópios, geradores de sinais, fontes de alimentação e multímetros foram substituídos, num total de 10 bancadas completas, que são utilizadas em aulas de diversas disciplinas como, por exemplo, Eletrônica Analógica, Eletrônica Digital, Instrumentação, Eletrônica de Potência, Microcontroladores e outras. Os alunos também contam com Laboratórios de Informática, Física, Química, Instalações Elétricas, Motores, Robótica, Automação, Eficiência Elétrica, Redes de Computadores entre outros.

Infelizmente nem todas as disciplinas da grade curricular do curso de Engenharia Eletrônica são agraciadas com aulas práticas, devido à falta de laboratórios específicos, como por exemplo algumas disciplinas na área de Telecomunicações. Nesse ponto que se enquadra a proposta desse artigo. Propomos a criação de um laboratório multidisciplinar, para uso nas disciplinas relacionadas às telecomunicações, baseado em Rádios Definidos por Software (RDS). Os RDSs são sistemas de comunicação onde parte do hardware comumente utilizado em sistemas semelhantes é substituído por softwares que são executados em computadores ou plataformas microprocessadas. Com essa arquitetura de Rádio Frequência (RF) configurável e programável é possível projetar, criar protótipos e implementar rapidamente sistemas de comunicação sem fio. 
A vantagem é a possibilidade de utilização da estrutura básica desse laboratório em aulas práticas de diversas disciplinas. Existem rádios definidos por software com arquiteturas e preços diversos e é possível dimensionar um laboratório que permita a realização de diversos experimentos práticos, de diversas disciplinas, sem onerar demasiadamente as contas da Universidade.

\section{RÁDIOS DEFINIDOS POR SOFTWARE}

Em um projeto financiado, que está atualmente em desenvolvimento na UPE, foram adquiridos alguns rádios definidos por software, bem como alguns amplificadores e antenas variadas, a serem utilizados em pesquisa que trata da comunicação entre veículos, com o envio de mensagens de veículo para veículo (V2V do inglês vehicle-to-vehicle) ou dos veículos para a infraestrutura montada nas cidades (V2I do inglês vehicle-to-infrastructure). O objetivo principal é minimizar o risco de colisões, aumentando a segurança no trânsito, mas também melhorar a trafegabilidade, otimizar o consumo de combustível e a emissão de poluentes através do traçado de rotas melhores e até, num futuro próximo, possibilitar a popularização dos veículos autônomos (FLANAGAN, 2018 e JAHNAVI, 2018).

No projeto, informações do veículo, tais como velocidade, rotação do motor, vazão nos bicos (consumo), tensão da bateria, dados sobre a injeção eletrônica, posicionamento da borboleta, entre outras, serão obtidas dos barramentos CAN (Controller Area Network) e/ou LIN (Local Interconnect Network) do veículo, através de um conector (ZELETIN, 2010 e LIU, 2018). A informação sobre a direção que o veículo trafega será obtida de um GPS integrado e, junto com as demais, encapsulada em um sistema microprocessado embarcado onde será executado o software de configuração e programação do rádio.

O rádio escolhido para ser embarcado no veículo foi um SDR de baixo custo do fabricante NUAND, modelo BladeRF x40, que pode ser visto na Figura 3 . Foram adquiridos quatro rádios, quatro amplificadores (para dar maior alcance à transmissão) e 4 antenas. Esse rádio é capaz de operar na faixa de $47 \mathrm{MHz}$ até $6 \mathrm{GHz}$ e custa $\bigcup \$ 420,00$. Sua escolha foi determinada pelo seu poder de processamento, sua faixa de frequências, sua compatibilidade com o software livre que será utilizado, seu pequeno peso $(250 \mathrm{~g})$ e dimensões reduzidas $(14 \mathrm{~cm} \times 10 \mathrm{~cm})$. A antena escolhida, do mesmo fabricante, foi uma antena Tri-band (que opera em 3 frequências) de uso geral, que custa $U \$ 25,00$. Respeitando-se as impedâncias, ela pode ser substituída por outras com maior ganho e diferentes diagramas de radiação. Também pesou na decisão o custo de, aproximadamente, $R \$ 3.000,00$ cada par rádio/antena, incluindo-se as taxas de importação. Para o projeto de comunicação veicular também foram adquiridos 4 amplificadores (mesmo fabricante) modelo XB-300, que permite um maior alcance das comunicações. Para uso didático, em laboratório, esses amplificadores não se fazem necessários.

O rádio escolhido para ficar nos pontos de apoio espalhados pela estrutura da cidade foi uma plataforma USRP (Universal Software Radio Peripheral) da National. O modelo escolhido foi o USRP 2901, totalmente compatível com o software livre a ser usado na programação. Como pode ser visto na Figura 4, esse rádio é um modelo bem mais completo que os rádios que foram escolhidos para serem embarcados nos veículos, consequentemente, mais caro. Foram adquiridos 3 desses para o projeto, a um custo de $\mathrm{R} \$ 12.500,00$ cada (setembro de 2020). 
Figura 3 - Rádios Nuand Blade RF X40.

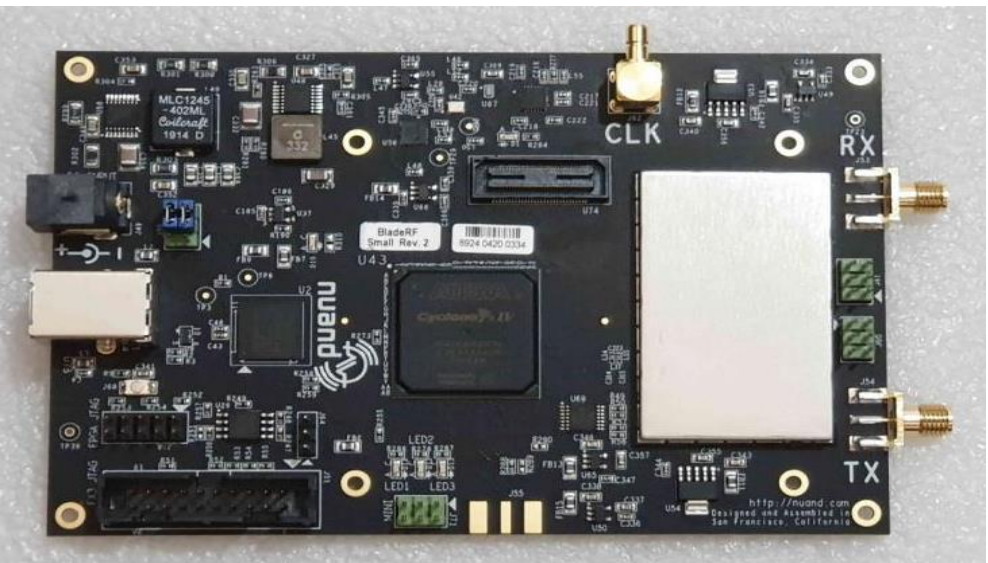

Fonte: Imagem do Autor.

Figura 4 - Rádio National USRP 2901 adquirido no projeto.

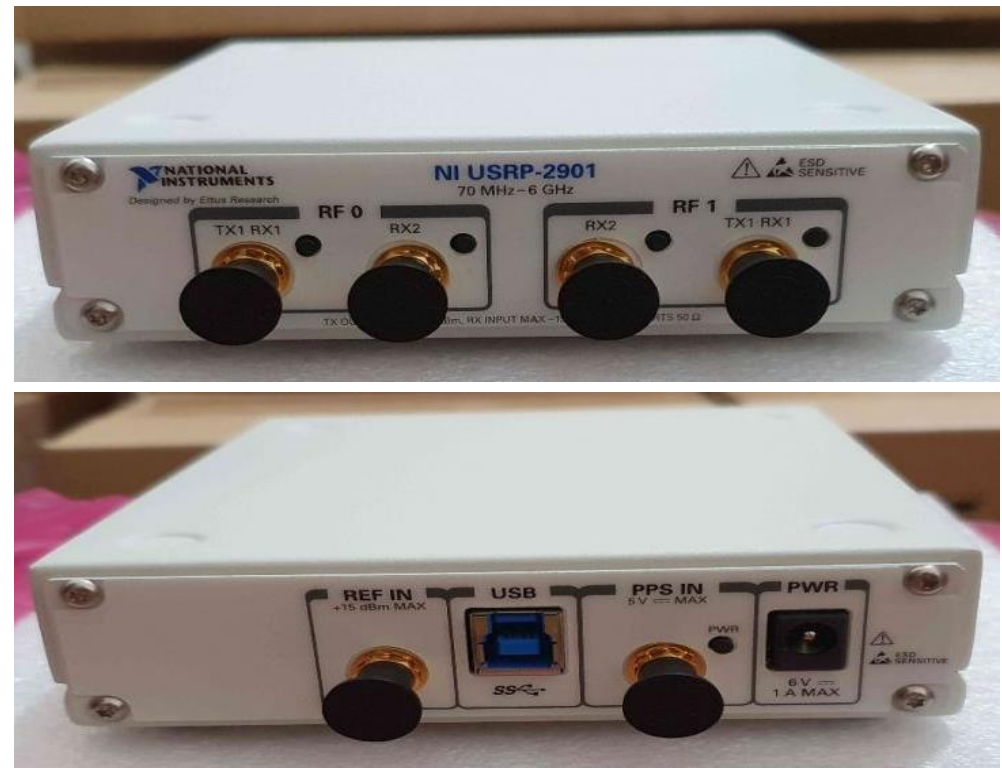

Fonte: Imagem do Autor.

Nos veículos é necessário ainda uma plataforma microprocessada, capaz de ler os dados de interesse nos barramentos CAN e/ou LIN, ler os dados do GPS, gerando o pacote a ser transmitido e, também, executar o software de programação do rádio. Para essa função foi escolhido um computador de placa única e tamanho reduzido, um Raspberry Pi. Já na infraestrutura montada em pontos da cidade o software do rádio pode ser executado tanto em uma placa Raspberry como num computador desktop ou num notebook.

O software escolhido, tão falado até agora, capaz de programar o rádio a executar em Software funções que tradicionalmente são executadas em Hardware, foi o GNU Radio (GURJÃO, 2013). É um kit de ferramentas de desenvolvimento gratuito que fornece blocos de processamento de sinais para implementar rádios e sistemas de comunicação (SELVA, 2011). 


\section{LABORATÓRIO DE TELECOMUNICAÇÕES BASEADO EM RADIOS DEFINIDOS POR SOFTWARE}

Com os conhecimentos e os equipamentos adquiridos ao longo do projeto de pesquisa sobre comunicação veicular, vislumbramos a possibilidade do uso da tecnologia dos Rádios Definidos por Software como uma forma de poder propiciar aos alunos de graduação em Engenharia Eletrônica da Escola Politécnica de Pernambuco, a realização de práticas que até o momento, não eram possíveis por falta de equipamentos para isso.

No Laboratório Didático Multidisciplinar de Telecomunicações não será necessário a aquisição dos computadores (ou plataformas de desenvolvimento microprocessadas como as placas Raspberry $\mathrm{Pi}$ ) pois as aulas ocorreriam em um dos diversos Laboratórios de Informática existentes na Universidade. Assim, nem um espaço físico definitivo seria necessário.

O uso dos rádios menores (Nuand ou similar) nas aulas traz, além das vantagens econômicas, maior portabilidade devido ao tamanho reduzido dos rádios. Assim, as aulas podem ser facilmente transferidas de um Laboratório de Informática para outro, caso necessário, bastando levar os rádios para o local desejado. Com o uso de notebooks, essas aulas podem ocorrer até em campo, onde pode ser estudado o alcance e a direcionalidade de diferentes antenas por exemplo. As aulas podem, inclusive, serem realizadas de forma remota, onde cada aluno pode realizar as práticas na sua residência, sendo as placas dos rádios disponibilizadas para os alunos por um certo período, num sistema de rodízio. Isso ajudaria bastante numa época de aulas remotas como a que estamos vivenciando.

O curso de Engenharia Eletrônica da Escola Politécnica da Universidade de Pernambuco possui 60 disciplinas obrigatórias e 34 eletivas. Analisando o currículo, selecionou-se as disciplinas onde o uso de Rádios Definidos por Software e do Software de configuração e simulação GNU Radio apresentaria melhorias na forma como as disciplinas são ofertadas, ajudando no aprendizado dos estudantes. As disciplinas elencadas são:

- Sinais e Sistemas - $6^{\circ}$ Período - Obrigatória

- Princípios de Comunicações - $7^{\circ}$ Período - Obrigatória

- Antenas - $10^{\circ}$ Período - Eletiva

- Comunicações Digitais - $10^{\circ}$ Período - Eletiva

- Comunicações Moveis - $10^{\circ}$ Período - Eletiva

- Criptografia - $10^{\circ}$ Período - Eletiva

- Filtros Analógicos e Digitais - $10^{\circ}$ Período - Eletiva

Com a grande evasão nos cursos de engenharia e a retenção dos alunos nos períodos iniciais, apenas cerca de $44 \%$ dos alunos chegam ao final do curso $(\mathrm{G} 1,2013)$. As disciplinas onde são ministrados os conteúdos referentes às Telecomunicações, devido aos pré-requisitos necessários, ocorrem a partir do $6^{\circ}$ Período do curso. A partir dessas informações, estima-se que o número de alunos nas turmas eletivas oscila entre 10 e 15. Esse número pode ser comprovado através do software de gestão acadêmica da Universidade. Já as turmas obrigatórias (Sinais e Sistemas e Princípios de Comunicações) são, geralmente, ofertadas em sua plenitude, e é com base nelas que dimensionamos 0 número de rádios necessários ao laboratório igual a 20.

Assim, considerando a aquisição de Rádios Nuand (por já termos as cotações devido ao projeto de comunicação veicular) que, com a antena de uso geral, custa $R \$ 3.000,00$. Considerando que não será necessário a aquisição de computadores por a Universidade já possuir vários laboratórios de informática. Considerando que o software para configuração dos rádios e simulação das comunicações é de uso livre e gratuito. Estimamos um custo 
de $R \$ 60.000,00$ para uma configuração mínima do laboratório. Se, acrescentarmos mais algumas antenas de ganho e direcionalidade diferentes, bem como materiais para confecção de antenas específicas com o uso de prototipadoras de circuitos impressos, o custo total do laboratório não excede $\mathrm{R} \$ 80.000,00$.

Esse é um valor bem aceitável, considerando que o laboratório pode ser utilizado por diversas disciplinas, onde poderão ser realizadas diversas práticas, além de os rádios poderem ser utilizados em pesquisas diversas. Com esse valor, dependendo do tamanho da turma, teremos praticamente um rádio por aluno! Mesmo que a turma seja maior, por sua portabilidade, os rádios podem ser usados num formato de rodízio. Não precisa cada aluno usar dois rádios, um para transmitir e outro para receber. Basta que, aos pares, os alunos estabeleçam a comunicação entre seus rádios. Como já pregava o educador e filósofo norte-americano John Dewey (1938), que inspirou no Brasil o movimento da Escola Nova, liderado por Anísio Teixeira, o processo prático potencializa as possibilidades e os resultados da educação, pelo envolvimento direto dos educandos (ROBERTS, 2019).

Vários trabalhos têm sido desenvolvidos sobre esse tema nos últimos anos. Vonehr, em 2016, realizou uma pesquisa para guiar os interessados em montar uma estrutura similar à que propomos aqui, para uso em cursos de telecomunicações. Comparou plataformas comerciais com base em parâmetros como custo, taxa de amostragem, faixa de frequências de operação. O rádio sugerido por eles, um HackRF-One apresenta características bem semelhantes ao que foi adquirido no projeto de comunicação veicular, mas o preço, apesar de ainda ser bastante inferior aos rádios da National, foi um diferencial na nossa escolha. O trabalho de Fortuna (2013), da UFPA, descreve a geração de alguns scripts a serem utilizados no ensino de sistemas de comunicação. Esse material tem sido disponibilizado de forma gratuita para a comunidade interessada em adotá-lo. Já Reis (2012), em São Paulo, mostra como o uso de arquitetura semelhante permite a rápida realização de experimentos, com menor risco de danificar os equipamentos (já que tudo é antes simulado no software), permitindo aos estudantes focarem seus esforços em, de fato, compreenderem os princípios das técnicas de telecomunicações. Welch (2012), não usou o GNU Radio. O rádio (USRP da National) foi configurado com o software LabVIEW, também da National, onde é criada uma instrumentação virtual que integrada ao rádio possibilita criar sistemas de comunicação em tempo real, a serem utilizados em aulas e pesquisas. O problema é o custo elevado, tanto do rádio como do software. Uma dissertação de mestrado da Universidade Federal de Uberlândia relata o desenvolvimento de sistema de processamento no GNU Radio, com o objetivo de aplicar uma Transformada Wavelet específica (Daubechies) na análise de sinais eletrocardiográficos e na compressão de arquivos (DINIZ, 2013).

\section{ANÁLISE DA APLICABILIDADE POR DISCIPLINAS}

A estrutura proposta, como já dito anteriormente, pode ser utilizada na realização de experimentos em diversas disciplinas ofertadas na graduação em engenharia eletrônica. A seguir apresentamos algumas práticas/aplicações dos rádios definidos por software e do software GNU Radio nas disciplinas (obrigatórias e eletivas) que fazem parte da linha do curso de eletrônica voltada as estudo das telecomunicações. Essa lista não é conclusiva, visto que também é possível a aplicação em outras disciplinas e/ou pesquisas não enumeradas aqui.

\subsection{Sinais e Sistemas}

O objetivo dessa disciplina é apresentar as ferramentas básicas da engenharia elétrica para representação, análise e tratamento de sinais. Os conteúdos compreendem a 
caracterização e operações de sinais e sistemas, as representações de Fourier, Laplace e $Z$ para sinais e sistemas de tempo contínuo e discreto. Tudo isso pode ser implementado no software GNU Radio, onde pode ser simulado a Função de Transferência de diversos sistemas e a resposta a estímulos como impulsos, degraus entre outros. O sistema representado no diagrama de fluxo de sinal (flowgraph) da Figura 5, por exemplo, realiza o truncamento da Série de Fourier de uma onda quadrada na sua $5^{\mathrm{a}}$ harmônica e o resultado pode ser facilmente visualizado através de blocos de instrumentação presentes no GNU Radio. Assim os alunos podem ver na prática os conceitos, às vezes abstratos, de que a soma de senos e cossenos resulta em ondas periódicas de formas diversas.

Figura 5 - Flowgraph, no GNU Radio, do truncamento da Série de Fourier de uma onda quadrada e sua saída.

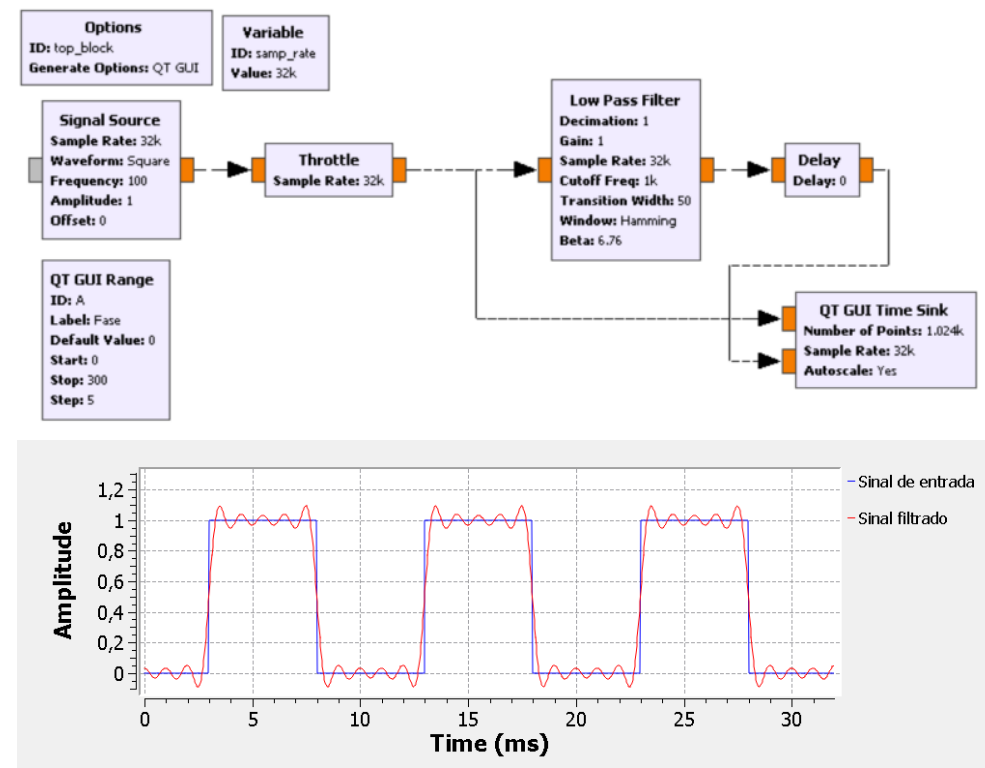

Fonte: Imagem do Autor.

\subsection{Princípios de Comunicações}

Uma das mais importantes disciplinas do curso de engenharia eletrônica e que serve como base para diversas disciplinas eletivas. Tem como objetivo introduzir os processos e procedimentos relacionados aos sistemas de comunicação. São apresentados diversos conceitos de filtragem e de modulação analógica. Os conteúdos estudados envolvem as redes lineares, modulação em amplitude (AM), frequência (FM), fase (PM), e a conversão analógico/digital por meio de modulações pulsadas. O emprego dos rádios e do GNU Radio podem proporcionar aos alunos uma melhor compreensão do conteúdo ministrado, permitindo que eles implementem estações de transmissão e recepção e estabeleçam uma comunicação real à distância. Operações, como a demodulação AM por exemplo, podem ser implementadas facilmente com o flowgraph indicado na Figura 6. Também é possível o aluno visualizar grandezas como a densidade espectral de potência que, em um laboratório convencional, só poderia ser analisada com equipamentos específicos como o Analisador de Espectro, cujo preço pode variar de algo em torno de U\$2.000,00 (Modelo SPA-921TG da empresa Com-Power) a até U\$120.000,00 (Modelo NI PXle-5668 da National). Um exemplo de aplicação dessa funcionalidade pode ser visualizado na Figura 7, onde temos o diagrama de fluxo de sinal de um sistema que exibe a análise espectral de uma onda quadrada antes e após ser filtrada por um filtro passa-baixas analógico, e também por um filtro passa-baixas digital do tipo FIR (Figura 8). Assim os alunos podem comparar a eficiência dos dois tipos de filtro. 
Figura 6 - Flowgraph de um demodulador AM, com seus sinais de entrada e saída.
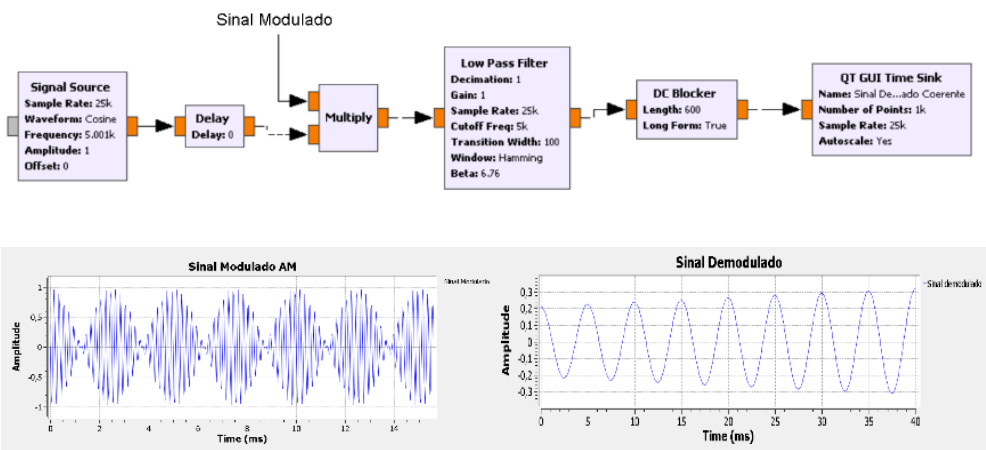

Fonte: Imagem do Autor.

Figura 7 - Flowgraph de sistema que exibe a análise espectral de uma onda quadrada antes e após filtro passa baixas analógico e digital (FIR).
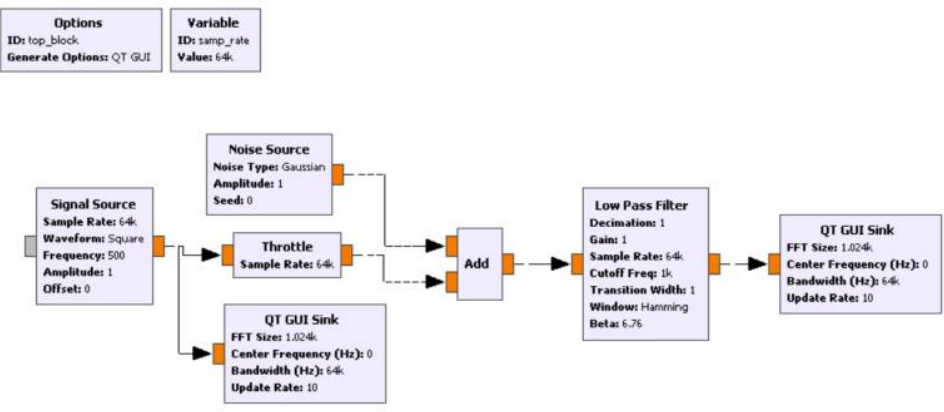

Fonte: Imagem do Autor.

Figura 8 - Análise espectral de uma onda quadrada antes e após filtro passa baixas analógico e digital (FIR).
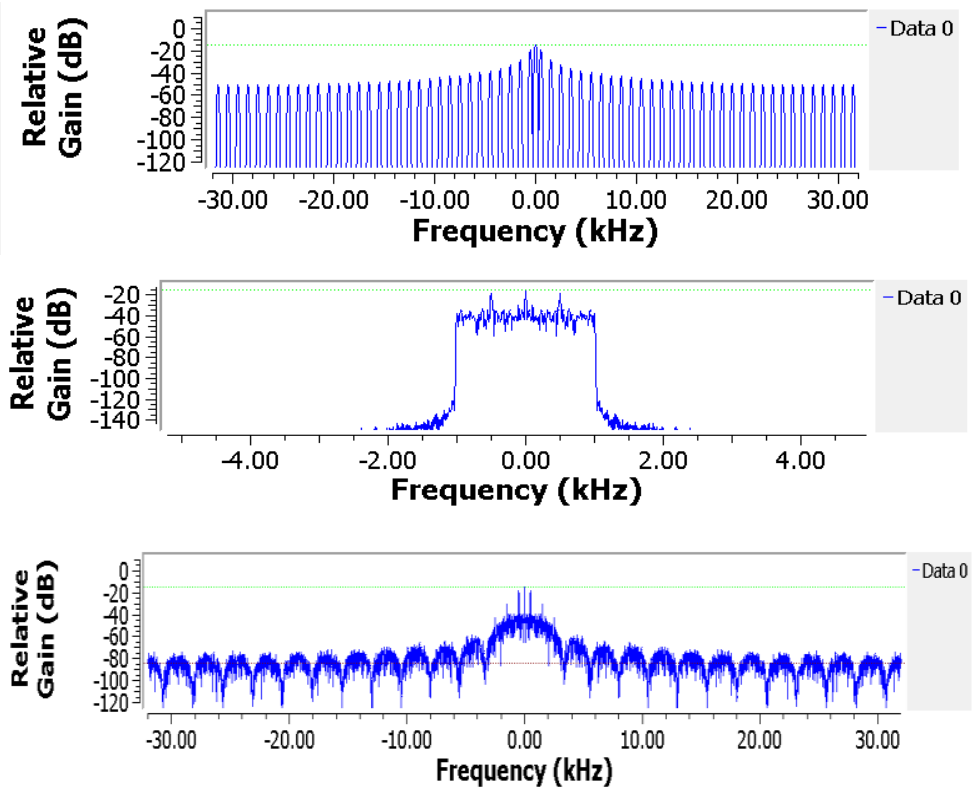

Fonte: Imagem do Autor. 


\subsection{Antenas}

Nessa disciplina os alunos aprendem conceitos básicos cobre propagação de ondas eletromagnéticas e a importância das antenas. A disciplina aborda tipos básicos de antenas, como projetá-las e técnicas de arranjo dessas antenas. Com a aquisição de outros tipos de antenas, como previsto no projeto do laboratório, os alunos podem realizar experimentos práticos com transmissão e recepção real, podendo observar o efeito de obstáculos no caminho, da distância, da direcionalidade e do ganho das antenas. Também é possível que os alunos projetem diferentes antenas, que podem ser confeccionadas por um serviço terceirizado. No projeto de comunicação foram adquiridas diversas e diferentes antenas. Na Figura 9, em sentido horário, podemos observar algumas das antenas adquiridas, como antenas direcionais Log Periodic PCB (uma na faixa de $400 \mathrm{MHz}$ a $1 \mathrm{GHz}$ e outra na faixa de $850 \mathrm{MHz}$ a $6.5 \mathrm{GHz}$ ), ambas com ganho de 5 a $6 \mathrm{dBi}$, antenas omnidirecionais verticais, com ganho de $3 \mathrm{dBi}$, e que operam em 2 faixas de frequências (2.4-2.5 e 4.9-5.9 GHz - Dualband), antenas omni-direcionais com mesmo ganho mas capazes de operar em 3 faixas de frequências (144 MHz, $400 \mathrm{MHz}, 1200 \mathrm{MHz}$ - Triband), entre outras.

Figura 9 - Exemplos de antenas adquiridas no projeto veicular, duas direcionais e duas omni-direcionais.
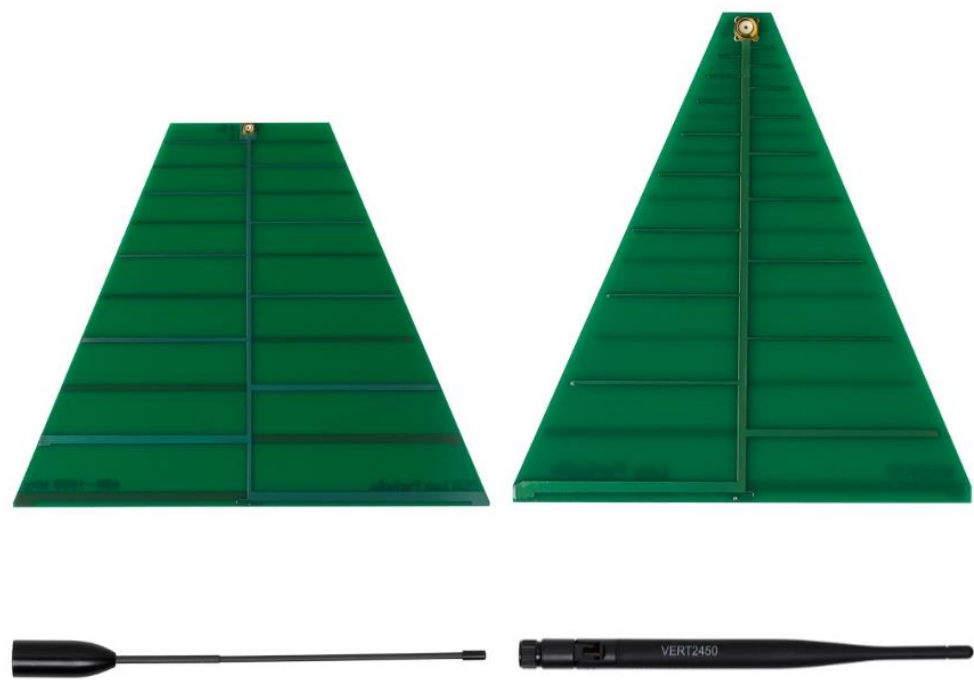

Fonte: Página da Ettus (https://www.ettus.com/product-categories/antennas/)

\subsection{Comunicações Digitais e Comunicações Móveis}

Nessas duas disciplinas eletivas é apresentado ao aluno uma introdução aos sistemas de comunicação digital. Técnicas de modulação, demodulação em banda base, noções sobre a ação dos ruídos nas comunicações, técnicas de multiplexação e múltiplo acesso, técnicas de codificação de sinais de voz e espalhamento espectral em redes móveis, bem como as evoluções e tendências tecnológicas, tais como GSM, WCDMA, LTE. O uso dos Rádios Definidos por Software, bem como do GNU Radio, traz para o aluno a possibilidade de conhecer de forma prática o que há de mais moderno com relação às técnicas de transmissão de dados. É possível, por exemplo, a implementação de uma rede de comunicação móvel padrão LTE (Long Term Evolution), que no Brasil ficou conhecida como rede 4G (4⿳亠口冋 Geração). No trabalho de Benkirane (2016) foi usado um rádio USRP da National para implementar uma modulação OFDM (Multiplexação por Divisão de Frequências Ortogonais), que distribui dados por um grande número de sub portadoras ortogonais. Trabalho semelhante foi realizado por Quispe, em 2017, também utilizando um rádio da National com o GNU Radio, e atestando a confiabilidade na transmissão por modulação OFDM, base das redes LTE. Na Figura 10 temos um exemplo que mostra como 
é simples o uso do GNU Radio para configurar o RDS como um sistema de modulação digital QAM (Quadrature Amplitude Modulation). A modulação de amplitude em quadratura é muito utilizada na transmissão de TV digital e de outros sistemas que necessitam de alta taxa de transferência. Na entrada foi colocado um sinal pseudo-aleatório de 100 amostras e com tamanho de 256 bits (dado a ser transmitido) e as saídas no domínio do tempo e da frequência são exibidas na Figura 11.

Figura 10 - Flowgraph de um modulador QAM com sequência pseudo-aleatória como entrada.

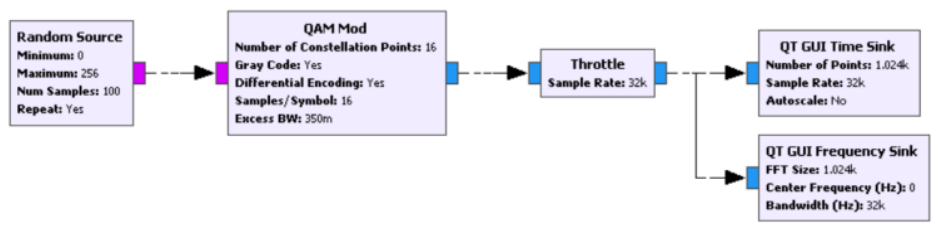

Fonte: Imagem do Autor.

Figura 11 - Saídas moduladas QAM no domínio do tempo e da frequência.
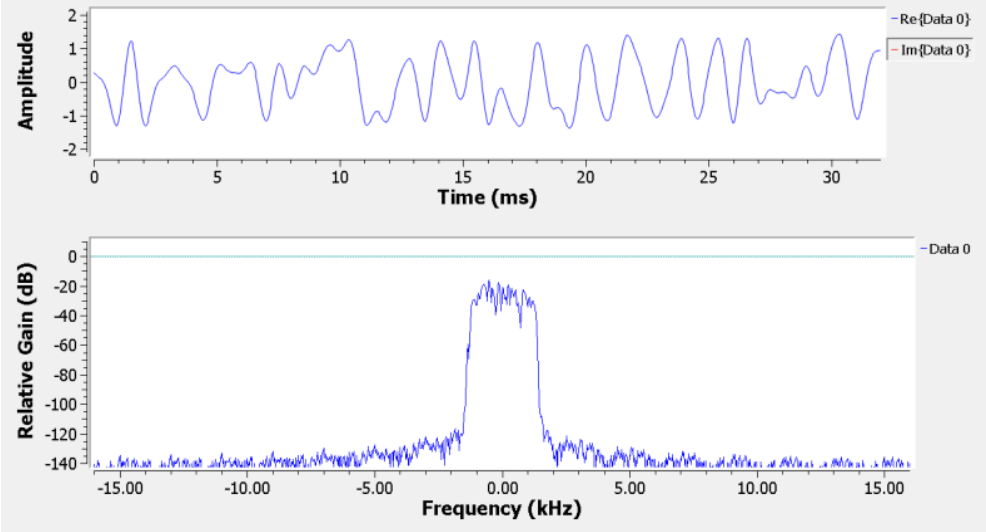

Fonte: Imagem do Autor.

\section{CONCLUSÕES}

Infelizmente o desmonte das universidades é uma realidade. A cada ano os investimentos dos governantes na educação vem caindo, obrigando os diretores e professores a ter um grande jogo de cintura. A pandemia pela qual estamos passando só veio agravar a situação. Por outro lado, precisamos prezar pela qualidade de ensino, capacitando os estudantes com o que há de mais atual no mercado de trabalho, para que eles possam ser logo inseridos e passem a ajudar a recuperação da economia. A proposta desse artigo não é algo inovador. Outros trabalhos já propuseram o uso de Rádios Definidos por Software como ferramenta em aulas na graduação. Mas a provocação desse presente artigo é mostrar o impacto dessa utilização, mostrando que o valor necessário para a implantação de um laboratório baseado nesses rádios não é nada exorbitante, e que o mesmo laboratório pode ser utilizado em diversas disciplinas e em várias linhas de pesquisa. O ensino prático, como pregado por diversos estudiosos, auxilia na compreensão e na retenção dos conhecimentos pelos estudantes. A aquisição de rádios mais simples, mais baratos, mas não menos eficientes, bem como a adoção de software livre, junto ao fato de, na maioria das instituições, já existirem laboratórios de informática, torna possível 
permitir aos alunos experiências inovadoras, de uma forma totalmente diferente da que eles estão acostumados.

\section{Agradecimentos}

O presente trabalho foi realizado com o apoio da Fundação de Amparo à Ciência e Tecnologia de Pernambuco (FACEPE), através do financiamento do Edital 11/2018, que aprovou o processo número APQ-0475-3.04/18 do qual este trabalho de pesquisa faz parte.

\section{REFERÊNCIAS}

ALFANO, B. e Niklas, J. Com o dobro de alunos, universidades federais têm mesma verba de 2004 e podem parar em julho. O Globo - Educação. Maio 2021. Disponível em: https://tinyurl.com/dobroalunos, Acesso em: 08 de maio de 2021.

BENKIRANE A. et al. Downlink implementation of the fourth generation of Wireless communication systems: The Long Term Evolution. Research Gate. Laboratoire Bordelais de Recherche en Informatique (LaBRI). 2016. Disponível em: https://tinyurl.com/LTEandUSRP. Acesso em: maio 2021.

DINIZ, P. C. A. Utilização de rádio definido por software para análise de sinais aplicados ao ensino em engenharia. 2013.

FLANAGAN, S. K., He, J. e Peng, X. H. Improving Emergency Collision Avoidance with Vehicle-to-Vehicle Communications, in 2018 IEEE 16th International Conference on Smart City; Exeter, United Kingdom, jun. 2018, p. 1322-1329.

FOLHA de São Paulo. Acompanhe a evolução de verbas repassadas às universidades federais do país. Folha de São Paulo Educação, São Paulo, 17 maio 2019. Disponível em: https://tinyurl.com/repasseUF. Acesso em: 01 maio de 2021.

FORTUNA, J. et al. Teaching DSP and Telecommunications with GNU Radio and Low Cost SDR Hardware. XXXI Simpósio Brasileiro de Telecomunicações - Sbrt 2013, setembro de 2013, Fortaleza, CE, 2013.

G1, Educação. Só $44 \%$ dos alunos de engenharia da última década terminaram o curso. São Paulo, julho 2013. Disponível em: https://tinyurl.com/so40termina. Acesso em: 09 maio 2021.

GURJÃO, E. C. Introdução ao GNU Radio. Revista de Tecnologia da Informação e Comunicação, Instituto de Estudos Avançados em Comunicações, Campina Grande, Paraíba. v. 3, n. 1, p. 1-5, 2013.

JAHNAVI, M. Vehicle to Vehicle Communication for Collision Avoidance, IJRASET, vol. 6, no 5, p. 1380-1386, maio de 2018, doi: 10.22214/ijraset.2018.5227.

LIU, Y. e Guo, J. Design of Improved Vehicle Collision Warning System Based on V2V Communication, in 2018 8th International Conference on Electronics Information and Emergency Communication (ICEIEC), Beijing, jun. 2018, p. 95-98. 
MORENO, Ana Carolina. $90 \%$ das universidades federais tiveram perda real no orçamento em cinco anos. G1 Educação, São Paulo, 29 junho 2018, Disponível em: https://tinyurl.com/verbaunivers. Acesso em: 01 maio de 2021.

QUISPE, J. L. et al. An SDR implementation of an OFDM transmitter and receiver in GNU Radio. VI Reunión de la Comunidad Científica Peruana en Brasil (CCP-BR). Campinas, SP, Brasil, junho de 2017.

REIS, A. L. et al. Software defined radio on digital communications: A new teaching tool. WAMICON 2012 IEEE Wireless \& Microwave Technology Conference. Anais. IEEE, 2012

ROBERTS, J. W. Beyond learning by doing: Theoretical currents in experiential education. Routledge, 2019. São Paulo. Julho 2013. Disponível em: https://tinyurl.com/evasaoengenharia. Acesso em: 04 maio de 2021.

SELVA, A. F. B. et al. Uma Introdução a SDR com GNU Radio. Anais do 2ำ Simpósio de Processamento de Sinais da UNICAMP, São Paulo, outubro 2011.

VONEHR, K.; NEUSON, W.; DUNNE, B. E. Software Defined Radio: Choosing the Right System for Your Communications Course. Proceedings of the American Society for Engineering Education, ASEE 123rd Annual Conference \& Exposition, New Orleans, 2016.

WELCH, T. B.; SHEARMAN, S. Teaching software defined radio using the USRP and LabVIEW. 2012 IEEE international conference on acoustics, speech and signal processing (ICASSP). Anais. IEEE, 2012

ZELETIN, R. P., Radusch, I. e Rigani, M. A., Vehicular-2-X communication: state-of-theart and research in mobile vehicular ad hoc networks. Springer Science \& Business Media, 2010.

\title{
SOFTWARE DEFINED RADIO AND GNU RADIO APPLIED TO UNDERGRADUATE COURSE IN ELECTRONIC ENGINEERING
}

\begin{abstract}
The continuous decrease of investments, by the government, in maintenance and creation of new university laboratories, makes it necessary to innovate. This work proposes creation of a telecommunications laboratory for the undergraduate course in electronics at Polithecnique School of the University of Pernambuco. Using simple and cheaper Software Defined Radios, together with the adoption of free software for configuration of radios and simulation of systems, allows the creation of environments where the student can learn, in practice, communication techniques. Previously, this was only taught theoretically. Learning by practicing helps in understanding and retaining knowledge. This article suggests a minimal setup for the laboratory and presents a budget estimate. In addition, it suggests different applications that can be developed in the various disciplines, mandatory or elective, offered in the course.
\end{abstract}

Keywords: Software Defined Radio, GNU Radio, Electronic Engineering, Telecommunications Laboratory. 